\title{
Model
}

\author{
Eyüb CANLI ${ }^{*}$, Ali ATEŞ${ }^{2}$, Şefik BiLíR ${ }^{3}$ \\ ${ }^{1}$ Selcuk University, Technology Faculty, Mechanical Engineering Department, Konya, Turkey. \\ ${ }^{2}$ Selcuk University, Ilgın V.H.S., Machinery and Metal Technologies Department, Konya, Turkey. \\ ${ }^{3}$ Konya Technical University, Faculty of Engineering and Natural Sciences, Mechanical Engineering Department, Konya, \\ Turkey.
}
* Corresponding author 1'e-mail: ecanli@selcuk.edu.tr ORCID ID: https://orcid.org/0000-0002-9358-1603
2e-mail:aates@selcuk.edu.tr ORCIDID: https://orcid.org/0000-0002-5506-8200
${ }^{3}$ e-mail: sbilir@ktun.edu.tr ORCID ID: https://orcid.org/0000-0001-7552-3437

Geliş Tarihi: 04.11.2020

Kabul Tarihi: 02.12.2020

\begin{tabular}{|c|c|}
\hline & \\
\hline $\begin{array}{c}\text { Keywords } \\
\text { Axisymmetric Flow; } \\
\text { Cylindrical } \\
\text { Coordinates; } k-\epsilon \\
\text { Turbulence Model; } \\
\text { Reynolds } \\
\text { Decomposition. }\end{array}$ & $\begin{array}{l}\text { In this work, equations that govern axisymmetric incompressible turbulent flow for heat transfer } \\
\text { calculations are derived assuming constant thermo-physical properties and a specific } \\
\text { nondimensionalization scheme. Vector algebra is used for expanding vector form of governing } \\
\text { equations in cylindrical coordinate system. Emphasis is on the derivatives of unit vectors according to } \\
\text { azimuthal direction. Reynolds decomposition is used for separating time averaged terms and Reynolds } \\
\text { Stress terms. Standard k- }- \text { turbulence model is selected for solving closure problem due to the Reynolds } \\
\text { stresses. Organization of the governing equations after model inputs is done explicitly. Also, parameters } \\
\text { that constitute nondimensionalization scheme are given. Evaluations of the complete process are given. } \\
\text { Two major aims of the work are presenting necessary equations explicitly and revealing some key steps } \\
\text { for reorganization of the equations. It is also aimed to present novel illustrations in order to contribute } \\
\text { comprehension of the concepts. }\end{array}$ \\
\hline
\end{tabular}

\section{Standart $k-\epsilon$ Modeli Temelinde Eksenel Simetrik Sıkıştırılamaz Türbülanslı Akış Isı Transferi için Boyutsuz Ana Denklemlerin Türetilmesi}

Öz

\begin{abstract}
Anahtar kelimeler
Eksenel Simetrik Akış;

Silindirik Koordinatlar;

$k-\epsilon$ Türbülans Modeli;

Reynolds Bileşenlerine

Ayırma.
\end{abstract}

\begin{abstract}
Bu çalışmada, sabit termo-fiziksel özellikler kabul edilerek ve belirli bir boyutsuzlaştırma şeması kullanılarak, ısı transferi hesaplamaları için eksenel simetrik sıkıştırılamaz türbülanslı akışı yöneten ana denklemler türetilmiştir. Silindirik koordinat sisteminde ana denklemlerin vektör formlarının açılması için vektör cebri kullanılmıştır. Açısal doğrultuya göre birim vektörlerin türevleri üzerine vurgulama yapılmıştır. Zaman ortalamalı terimler ile Reynolds gerilmeleri terimlerinin ayrılması için Reynolds bileşenlerine ayırma yöntemi kullanılmıştır. Reynolds gerilmelerinden kaynaklanan kapama sorununu çözmek için standart k- $\epsilon$ türbülans modeli seçilmiştir. Model girdilerinden sonra ana denklemlerin düzenlenmesi açık olarak verilmiştir. Ayrıca boyutsuzlaştırma şemasını oluşturan parametreler verilmiştir. Bütün sürecin değerlendirmeleri verilmiştir. Çalışmanın iki ana amacı, gerekli denklemlerin açık olarak verilmesi ve denklemlerin düzenlenmesindeki bazı anahtar adımların ortaya konmasıdır. Ayrıca kavramların anlaşılmasına katkı sağlamak için özgün görsellerin sunumu amaçlanmıştır.
\end{abstract}

(C) Afyon Kocatepe Üniversitesi

\section{Introduction}

Coordinate systems for spatial resolution of a flow field are very important in fluid mechanics and computational fluid dynamics (CFD). In finite volume method, for instance, variables are integrated over the control volumes according to the coordinate system. Although coordinate systems are not changing the ultimate and correct results after a 
computational process, process itself can become chaotic with selection of a specific coordinate system and related approach. Literature has frequently uses index notation abbreviations or shorthand notations. Also, the basic examples are mostly on Cartesian coordinates. Therefore, in this work, an axisymmetric flow field in two dimensional cylindrical coordinates is considered for derivation of flow, turbulence modeling and energy equations from conservation laws in vector forms.

Governing equations in fluid mechanics are well established over nearly two centuries. Their background is based on physics by conservation laws and they are expressed by mathematical models. These mathematical expressions are axioms. In other words, they cannot be validated mathematically but they are known. However, mathematic itself has a language and sometimes this language has differences in symbols for different fields. This changing mathematical alphabet sometimes leads to vast amount of writings for a single statement. In order to overcome this cumbersome situation, literature developed kind of abbreviation by notations such as "shorthand" notations, index notations and vector operators. As a side effect, users of this language can be mistaken for a step while extracting the compact notation to reach original statement. Equations of fluid mechanics are used and investigated by many disciplines and practitioners including mathematicians, physicists, and engineers. Accordingly, symbols, expressions, methods, applications and approaches differ and constitute a widespread field. Therefore, people dealing with fluid mechanics, especially people from industry and new practitioners can have problems for, let's say, special arrangements such as coordinate systems. An example can be given as Computational Fluid Dynamics (CFD) governing equations in cylindrical coordinate systems.

Cylindrical coordinates offer advantages for certain conditions and geometries such as axisymmetric flows, i.e. pipes, nozzles and such. A cylindrical surface can be defined only by changes in coordinates and no additional operations are needed for simple geometries. However, additional terms emerge in governing equations. Most of these terms are due to growing arc in positive radial direction, as it leads to a growing surface as in pipes. Nevertheless, finding or recognizing these extra terms is not straightforward. The main reason is using mathematical procedure, not physical reasoning, and missing details of operators and notations special to the coordinate system. Even so, correct form of operators can correct a lot of errors, in the absence of physical reasoning. Considering the capacity of modern literature resources, citable literature contributions about explicit mathematical expressions and their derivation processes can reduce mistakes.

The major problem associated with deriving CFD governing equations for cylindrical coordinates is examples in reference books. Most of them are for Cartesian coordinates. Some resources use index notation or Einstein notation for abbreviation. However, preparing a recipe to extract equations in index notations to cylindrical coordinates is not seen logical and haven't been encountered. Writing equations in cylindrical coordinates using vector forms seems to be the most appropriate one but one should pay attention to use correct operators because operators and operations change according to the coordinate systems. The final tool can be General Orthogonal Curvilinear Coordinates (GOCC). This tool can be very useful but still needs to have knowledge about vector operations. And sometimes become cumbersome. Also, it is hard to find all equation relations, especially for turbulent model equations.

This work uses some well-known references alongside of new ones in order to write conservation expressions in cylindrical coordinates. Conservation expressions in vector form are written from Moukalled et al. (2016). This book is relatively new and includes computer codes for MATLAB and OpenFOAM. It also gives vector operations in necessary amount for fluid dynamics. Book of Anderson et al. is used for GOCC and Reynolds Decomposition (RD) (Anderson et al. 1984). For GOCC, book of Happel and Brenner is a more comprehensive resource (Happel and Brenner 2012). Books of Versteeg and Malalasekera (2007), Patankar (1980) and Tennekeys and Lumley (1972) are used for the general frame. Of course, well 
known reference of Launder and Spalding (1974) is used for $\mathrm{k}-\epsilon$ method.

In this work, governing equations are derived from vector forms by using vector operations for heat transfer and turbulent fluid flow of an incompressible fluid in 2D axisymmetric domain and cylindrical coordinates. RD is performed and equations are then written in non-dimensional form. Vector operations and simplifications special to the cylindrical coordinates are emphasized. Implications relating to the work are evaluated. A citable document is aimed for related audience by presenting explicit forms of equations and operations.

\section{Theory}

\subsection{Expanding vector forms}

All symbols in the paper are given in a nomenclature after references section. Vectors are written in straight font while scalars are written italic. Also, parenthesis changes according to content. Round parenthesis (), square brackets [] and braces \{\} are used for scalars, vectors and tensors respectively. In the following, continuity, momentum and energy equations in vector form are given between (1)-(3) respectively.

Vector dot product (shown with $\sqcup$ symbol) is a special vector algebra operation. By vector dot product, vector components of one vector are multiplied with their corresponding parts of another vector. The dot product of two vectors returns a scalar.

$\frac{\partial \rho}{\partial t}+\nabla[\rho \mathrm{v}]=0$

$\frac{\partial}{\partial t}[\rho \mathrm{v}]+\nabla \square\{\rho \mathrm{vv}\}=\mathrm{f}$

$\frac{\partial}{\partial t}(\rho e)+\nabla[\rho v e]=$

$-\nabla \llbracket \dot{q}_{s}-\nabla[\rho \mathrm{v}]+\nabla[\tau \llbracket \mathrm{v}]+\mathrm{f}_{b}\left[\mathrm{v}+\dot{q}_{V}\right.$

For incompressible fluid, continuity equation becomes; $\nabla \sqsubset \mathrm{v}=0$

Force term of equation (2) can be expressed as (5) in the absence of gravity and other external sources.

$\mathrm{f}_{s}=-\nabla p+[\nabla \square \tau]$

(5) contains surface forces in the absence of body forces.

Shear stress tensor can be expressed in (6) for Newtonian fluids.

$\tau=\mu\left\{\nabla \mathrm{v}+(\nabla \mathrm{v})^{\mathrm{T}}\right\}+\lambda(\nabla \sqsubset \mathrm{v}) \mathrm{I}$

Divergence of $\tau$ is as following.

$\left[\nabla[\tau]=\nabla\left[\mu\left(\nabla \mathrm{v}+(\nabla \mathrm{v})^{\mathrm{T}}\right)\right]+\nabla(\lambda \nabla[\mathrm{v})\right.$

When (4) is used with (6) and (7) in (2) and viscosity is assumed constant, the equation has the following form.

$\frac{\partial}{\partial t}[\rho \mathrm{v}]+\nabla\left[\{\rho \mathrm{vv}\}=-\nabla p+\mu \nabla^{2} \mathrm{v}\right.$

In energy equation, temperature is used in most studies as a primitive variable. Therefore, energy equation is modified as shown in (9) to have a form in terms of temperature.

$c_{p}\left[\frac{\partial}{\partial t}(\rho T)+\nabla[\rho \mathrm{v} T]\right]=\nabla[k \nabla T]$

$-\left(\frac{\partial(\ln \rho)}{\partial(\ln T)}\right)_{p} \frac{D p}{D t}+(\tau: \nabla \mathrm{v})+\dot{q}_{V}$

Double dot product in (9) produces viscous dissipation. With constant viscosity assumption, incompressibility and no additional heat generation, (9) reduces to (10).

$\frac{\partial}{\partial t}\left(\rho c_{p} T\right)+\nabla\left[\rho c_{p} \mathrm{v} T\right]=\nabla[k \nabla T]+\Phi$

In order to extract equations (1)-(10) in cylindrical coordinates, operators and operations should be written in cylindrical coordinates. To do so, required vector operator and operations are given in Table 1.

Table 1. List of required vectors, tensors, operators and operations.

\begin{tabular}{cccc}
\hline Vectors & Tensors & Operators & Operations \\
V & $\tau$ & $\nabla$ & $\sqcup$ \\
- & $\{\mathrm{vv}\}$ & $\nabla^{2}$ & $:$ \\
- & - & - & $\times$ \\
\hline
\end{tabular}


Velocity vector in cylindrical coordinates is given below.

$\mathrm{v}=u \hat{\mathrm{e}}_{x}+v \hat{\mathrm{e}}_{r}+w \hat{\mathrm{e}}_{\theta}$

Del operator can be converted from Cartesian coordinates to cylindrical coordinates by below procedure between (12) and (23).

$\nabla=\hat{\mathrm{e}}_{x} \frac{\partial}{\partial x}+\hat{\mathrm{e}}_{y} \frac{\partial}{\partial y}+\hat{\mathrm{e}}_{z} \frac{\partial}{\partial z}$

Comparison of Cartesian and cylindrical coordinates is given in Figure 1 in a schematic manner. This figure does not only give axes but also compares spaces.

Conversion of the coordinates follows below steps:

$r=\sqrt{z^{2}+y^{2}}$

$\theta=\tan ^{-1} \frac{y}{z}$

$\frac{\partial r}{\partial z}=\frac{2 z}{2 \sqrt{z^{2}+y^{2}}}=\frac{z}{\sqrt{z^{2}+y^{2}}}=\frac{z}{r}=\frac{r \cos \theta}{r}=\cos \theta$

$\frac{\partial r}{\partial y}=\frac{2 y}{2 \sqrt{z^{2}+y^{2}}}=\frac{y}{\sqrt{z^{2}+y^{2}}}=\frac{y}{r}=\frac{r \sin \theta}{r}=\sin \theta$

$\frac{\partial \theta}{\partial z}=\frac{1}{1+\frac{y^{2}}{z^{2}}} \frac{-y}{z^{2}}=\frac{z^{2}}{z^{2}+y^{2}} \frac{-y}{z^{2}}$

$=\frac{-y}{z^{2}+y^{2}}=\frac{-r \sin \theta}{r^{2}}=\frac{-\sin \theta}{r}$

$\frac{\partial \theta}{\partial y}=\frac{1}{1+\frac{y^{2}}{z^{2}}} \frac{z}{z^{2}}=\frac{z^{2}}{z^{2}+y^{2}}=\frac{r \cos \theta}{r^{2}}=\frac{\cos \theta}{r}$

$\frac{\partial}{\partial z}=\frac{\partial r}{\partial z} \frac{\partial}{\partial r}+\frac{\partial \theta}{\partial z} \frac{\partial}{\partial \theta}$

$\frac{\partial}{\partial y}=\frac{\partial r}{\partial y} \frac{\partial}{\partial r}+\frac{\partial \theta}{\partial y} \frac{\partial}{\partial \theta}$

$\hat{\mathrm{e}}_{z}=\cos \theta \hat{\mathrm{e}}_{r}+\sin \theta \hat{\mathrm{e}}_{\theta}$

$\hat{\mathrm{e}}_{y}=\sin \theta \hat{\mathrm{e}}_{r}+\cos \theta \hat{\mathrm{e}}_{\theta}$

$$
\begin{aligned}
& \nabla=\mathrm{i} \frac{\partial}{\partial x}+\left(\sin \theta \hat{\mathrm{e}}_{r}+\cos \theta \hat{\mathrm{e}}_{\theta}\right)\left(\frac{\partial r}{\partial y} \frac{\partial}{\partial r}+\frac{\partial \theta}{\partial y} \frac{\partial}{\partial \theta}\right) \\
& +\left(\cos \theta \hat{\mathrm{e}}_{r}-\sin \theta \hat{\mathrm{e}}_{\theta}\right)\left(\frac{\partial r}{\partial z} \frac{\partial}{\partial r}+\frac{\partial \theta}{\partial z} \frac{\partial}{\partial \theta}\right) \\
& \nabla=\mathrm{i} \frac{\partial}{\partial x}+\left(\sin \theta \hat{\mathrm{e}}_{r}+\cos \theta \hat{\mathrm{e}}_{\theta}\right)\left(\sin \theta \frac{\partial}{\partial r}+\frac{\cos \theta}{r} \frac{\partial}{\partial \theta}\right) \\
& +\left(\cos \theta \hat{\mathrm{e}}_{r}-\sin \theta \hat{\mathrm{e}}_{\theta}\right)\left(\cos \theta \frac{\partial}{\partial r}-\frac{\sin \theta}{r} \frac{\partial}{\partial \theta}\right) \\
& \nabla=\mathrm{i} \frac{\partial}{\partial x}+\sin { }^{2} \theta \hat{\mathrm{e}}_{r} \frac{\partial}{\partial r}+\sin \theta \frac{\cos \theta}{r} \hat{\mathrm{e}}_{r} \frac{\partial}{\partial \theta} \\
& +\cos \theta \sin \theta \hat{\mathrm{e}}_{\theta} \frac{\partial}{\partial r}+\cos \theta \frac{\cos \theta}{r} \hat{\mathrm{e}}_{\theta} \frac{\partial}{\partial \theta} \\
& +\cos { }^{2} \theta \hat{\mathrm{e}}_{r} \frac{\partial}{\partial r}+\cos \theta \frac{-\sin \theta}{r} \hat{\mathrm{e}}_{r} \frac{\partial}{\partial \theta} \\
& -\sin \theta \cos \theta \hat{\mathrm{e}}_{\theta} \frac{\partial}{\partial r}-\sin \theta \frac{-\sin \theta}{r} \hat{\mathrm{e}}_{\theta} \frac{\partial}{\partial \theta} \\
& \nabla=\hat{\mathrm{e}}_{x} \frac{\partial}{\partial x}+\hat{\mathrm{e}}_{r} \frac{\partial}{\partial r}+\frac{1}{r} \hat{\mathrm{e}}_{\theta} \frac{\partial}{\partial \theta}
\end{aligned}
$$

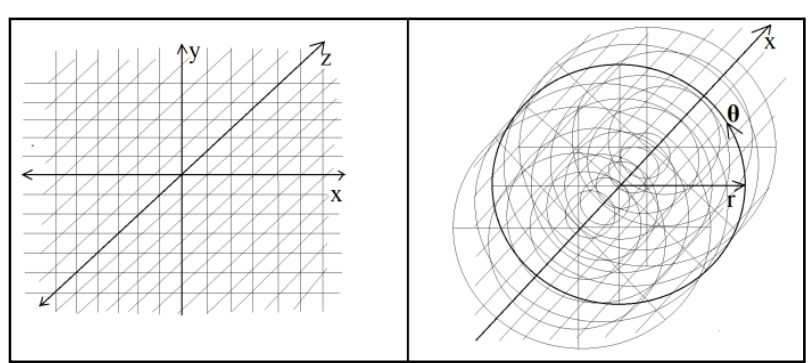

Figure 1. Schematic comparison of Cartesian and cylindrical coordinate systems.

Writing del operator in cylindrical coordinates is very crucial because additional terms in governing equations emerge from partial derivation of azimuthal and radial unit vectors according to azimuthal direction. By mathematical expression; $\frac{\partial}{\partial \theta} \hat{\mathrm{e}}_{r}=\hat{\mathrm{e}}_{\theta}$ and $\frac{\partial}{\partial \theta} \hat{\mathrm{e}}_{\theta}=-\hat{\mathrm{e}}_{r}$.

According to vector dot operation principle and del operator in cylindrical coordinates, continuity can be written as:

$\nabla \sqsubset v=\frac{\partial u}{\partial x}+\frac{v}{r}+\frac{1}{r} \frac{\partial w}{\partial \theta}+\frac{\partial v}{\partial r}=0$

Newly emerging terms in del operator creates additional terms in continuity for cylindrical 
coordinates. This is mainly due to the derivation according to the azimuthal direction.

The second term in (8) is divergence of a tensor and forms a vector.

$$
\begin{aligned}
& \nabla \underline{\{} \rho \mathrm{vv}\}=\left[\hat{\mathrm{e}}_{x} \frac{\partial}{\partial x}+\hat{\mathrm{e}}_{\theta} \frac{1}{r} \frac{\partial}{\partial \theta}+\hat{\mathrm{e}}_{r} \frac{\partial}{\partial r}\right] \\
& \left\{\begin{array}{l}
\hat{\mathrm{e}}_{x} \hat{\mathrm{e}}_{x} \rho u u+\hat{\mathrm{e}}_{x} \hat{\mathrm{e}}_{r} \rho u v+\hat{\mathrm{e}}_{x} \hat{\mathrm{e}}_{\theta} \rho u w \\
+\hat{\mathrm{e}}_{r} \hat{\mathrm{e}}_{x} \rho v u+\hat{\mathrm{e}}_{r} \hat{\mathrm{e}}_{r} \rho v v+\hat{\mathrm{e}}_{r} \hat{\mathrm{e}}_{\theta} \rho v w \\
+\hat{\mathrm{e}}_{\theta} \hat{\mathrm{e}}_{x} \rho w u+\hat{\mathrm{e}}_{\theta} \hat{\mathrm{e}}_{r} \rho w v+\hat{\mathrm{e}}_{\theta} \hat{\mathrm{e}}_{\theta} \rho w w
\end{array}\right\}
\end{aligned}
$$

When special care is paid on partial derivation of azimuthal and radial unit vectors according to azimuthal direction, (25a) can be written as (25b).

$\nabla\{\rho \mathrm{vv}\}=\hat{\mathrm{e}}_{x}\left[\begin{array}{l}\frac{\partial}{\partial x} \rho u u+\frac{\partial}{\partial r} \rho u v+\frac{\rho w}{r} \frac{\partial}{\partial \theta} u \\ +\frac{u}{r} \frac{\partial}{\partial \theta} \rho w+\frac{\rho u v}{r}\end{array}\right]$

$+\hat{\mathrm{e}}_{r}\left[\begin{array}{l}\frac{\partial}{\partial x} \rho u v+\frac{\partial}{\partial r} \rho v v+\frac{\rho w}{r} \frac{\partial}{\partial \theta} v+\frac{v}{r} \frac{\partial}{\partial \theta} \rho w \\ +\frac{\rho v v}{r}-\frac{r w^{2}}{r}\end{array}\right]$

$+\hat{\mathrm{e}}_{\theta}\left[\begin{array}{l}\frac{\partial}{\partial x} \rho u w+\frac{\rho w v}{r}+\frac{\rho w v}{r}+\frac{\rho w}{r} \frac{\partial}{\partial \theta} w \\ +\frac{w}{r} \frac{\partial}{\partial \theta} \rho w+\frac{\partial}{\partial r} \rho v w\end{array}\right]$

For the last term of (8), Laplacian operator should be written in cylindrical coordinates. This can be done by dot product of two del operators.

$$
\begin{aligned}
& \nabla \square \nabla=\left[\hat{\mathrm{e}}_{x} \frac{\partial}{\partial x}+\hat{\mathrm{e}}_{r} \frac{\partial}{\partial r}+\frac{1}{r} \hat{\mathrm{e}}_{\theta} \frac{\partial}{\partial \theta}\right] \\
& {\left[\hat{\mathrm{e}}_{x} \frac{\partial}{\partial x}+\hat{\mathrm{e}}_{r} \frac{\partial}{\partial r}+\frac{1}{r} \hat{\mathrm{e}}_{\theta} \frac{\partial}{\partial \theta}\right]} \\
& \nabla^{2}=\frac{\partial^{2}}{\partial x^{2}}+\frac{\partial^{2}}{\partial r^{2}}+\frac{1}{r^{2}} \frac{\partial^{2}}{\partial \theta^{2}}+\frac{1}{r} \frac{\partial}{\partial r}
\end{aligned}
$$

Del operator itself is a vector. Therefore, dot product of a vector with del operator means a divergence and forms a scalar. Accordingly, Laplacian operator is a scalar operator. However, this scalar operator still contains partial derivative according to azimuthal direction and therefore yields additional terms comparing to its Cartesian counterpart. Accordingly, the last term of (8) can be expanded as following.

$$
\mu \nabla^{2} \mathrm{v}=\mu\left[\begin{array}{l}
\left(\frac{\partial^{2}}{\partial x^{2}}+\frac{\partial^{2}}{\partial r^{2}}+\frac{1}{r^{2}} \frac{\partial^{2}}{\partial \theta^{2}}+\frac{1}{r} \frac{\partial}{\partial r}\right) \\
{\left[u \hat{\mathrm{e}}_{x}+v \hat{\mathrm{e}}_{r}+w \hat{\mathrm{e}}_{\theta}\right]}
\end{array}\right]
$$

$$
\mu \nabla^{2} \mathrm{v}=\mu\left[\begin{array}{l}
\hat{\mathrm{e}}_{x} \frac{\partial^{2}}{\partial x^{2}} u+\hat{\mathrm{e}}_{x} \frac{\partial^{2}}{\partial r^{2}} u+\hat{\mathrm{e}}_{x} \frac{1}{r^{2}} \frac{\partial^{2}}{\partial \theta^{2}} u \\
+\hat{\mathrm{e}}_{x} \frac{1}{r} \frac{\partial}{\partial r} u+\hat{\mathrm{e}}_{r} \frac{\partial^{2}}{\partial x^{2}} v+\hat{\mathrm{e}}_{r} \frac{\partial^{2}}{\partial r^{2}} v \\
+\frac{\partial}{\partial \theta}\left(\frac{\partial}{\partial \theta} v \hat{\mathrm{e}}_{r}\right)+\hat{\mathrm{e}}_{r} \frac{1}{r} \frac{\partial}{\partial r} v \\
+\hat{\mathrm{e}}_{\theta} \frac{\partial^{2}}{\partial x^{2}} w+\hat{\mathrm{e}}_{\theta} \frac{\partial^{2}}{\partial r^{2}} w+\hat{\mathrm{e}}_{\theta} \frac{1}{r} \frac{\partial}{\partial r} w \\
+\frac{1}{r^{2}} \frac{\partial}{\partial \theta}\left(\frac{\partial}{\partial \theta} w \hat{\mathrm{e}}_{\theta}\right)
\end{array}\right]
$$

$$
\mu \nabla^{2} \mathbf{v}=\mu\left[\begin{array}{c}
\hat{\mathrm{e}}_{x}\left(\begin{array}{c}
\frac{\partial^{2} u}{\partial x^{2}}+\frac{\partial^{2} u}{\partial r^{2}} \\
+\frac{1}{r^{2}} \frac{\partial^{2} u}{\partial \theta^{2}}+\frac{1}{r} \frac{\partial u}{\partial r}
\end{array}\right) \\
+\hat{\mathrm{e}}_{r}\left(\begin{array}{c}
\frac{\partial^{2} v}{\partial x^{2}}+\frac{\partial^{2} v}{\partial r^{2}}+\frac{1}{r} \frac{\partial v}{\partial r} \\
+\frac{1}{r^{2}} \frac{\partial^{2} v}{\partial \theta^{2}}-\frac{v}{r^{2}} \\
-\frac{1}{r^{2}} \frac{\partial w}{\partial \theta}-\frac{1}{r^{2}} \frac{\partial w}{\partial \theta}
\end{array}\right) \\
+\left(\begin{array}{c}
\frac{\partial^{2} w}{\partial x^{2}}+\frac{\partial^{2} w}{\partial r^{2}}+\frac{1}{r} \frac{\partial w}{\partial r} \\
+\frac{1}{r^{2}} \frac{\partial v}{\partial \theta}+\frac{1}{r^{2}} \frac{\partial v}{\partial \theta} \\
+\frac{1}{r^{2}} \frac{\partial^{2} w}{\partial \theta^{2}}-\frac{w}{r^{2}}
\end{array}\right)
\end{array}\right]
$$

At this phase, conservation of momentum can be divided into three considering its components in three directions. Nevertheless, three equations seem very complicated and can be reorganized in order to obtain a simpler form by using (24). When left side of the three momentum equations are rearranged, (24) appears in them and since (24) is equal to " 0 ", those terms vanish.

Final forms are: 
$x$-momentum;

$$
\begin{aligned}
& \frac{\partial u}{\partial t}+u \frac{\partial u}{\partial x}+v \frac{\partial u}{\partial r}+\frac{w}{r} \frac{\partial u}{\partial \theta}=-\frac{1}{\rho} \frac{\partial p}{\partial x} \\
& +v\left(\frac{\partial^{2} u}{\partial x^{2}}+\frac{\partial^{2} u}{\partial r^{2}}+\frac{1}{r^{2}} \frac{\partial^{2} u}{\partial \theta^{2}}+\frac{1}{r} \frac{\partial u}{\partial r}\right)
\end{aligned}
$$

r-momentum;

$$
\begin{aligned}
& \frac{\partial v}{\partial t}+u \frac{\partial v}{\partial x}+v \frac{\partial v}{\partial r}+\frac{w}{r} \frac{\partial v}{\partial \theta}-\frac{w^{2}}{r} \\
& =-\frac{1}{\rho} \frac{\partial p}{\partial r}+v\left(\begin{array}{l}
\frac{\partial^{2} v}{\partial x^{2}}+\frac{\partial^{2} v}{\partial r^{2}}+\frac{1}{r^{2}} \frac{\partial^{2} v}{\partial \theta^{2}} \\
+\frac{1}{r} \frac{\partial v}{\partial r}-\frac{2}{r^{2}} \frac{\partial w}{\partial \theta}-\frac{v}{r^{2}}
\end{array}\right)
\end{aligned}
$$

\section{$\vartheta$-momentum;}

$$
\begin{aligned}
& \frac{\partial w}{\partial t}+u \frac{\partial w}{\partial x}+v \frac{\partial w}{\partial r}+\frac{w}{r} \frac{\partial w}{\partial \theta}+\frac{w v}{r} \\
& =-\frac{1}{\rho} \frac{1}{r} \frac{\partial p}{\partial \theta}+v\left(\begin{array}{l}
\frac{\partial^{2} w}{\partial x^{2}}+\frac{\partial^{2} w}{\partial r^{2}}+\frac{1}{r^{2}} \frac{\partial^{2} w}{\partial \theta^{2}} \\
+\frac{1}{r} \frac{\partial w}{\partial r}+\frac{2}{r^{2}} \frac{\partial v}{\partial \theta}-\frac{w}{r^{2}}
\end{array}\right)
\end{aligned}
$$

A scalar is obtained when vector operations are applied on energy equation (10). The final term, viscous dissipation, should be written from $(\tau: \nabla v)$. In order to extract (6), a tensor is formed as in (29).

$$
\begin{aligned}
& \{\nabla \mathrm{v}\}=\left\{\left[\hat{\mathrm{e}}_{x} \frac{\partial}{\partial x}+\hat{\mathrm{e}}_{\theta} \frac{1}{r} \frac{\partial}{\partial \theta}+\hat{\mathrm{e}}_{r} \frac{\partial}{\partial r}\right]\left[u \hat{\mathrm{e}}_{x}+w \hat{\mathrm{e}}_{\theta}+v \hat{\mathrm{e}}_{r}\right]\right\} \\
& \{\nabla \mathrm{v}\}=\left\{\begin{array}{lcc}
\frac{\partial u}{\partial x} & \frac{\partial v}{\partial x} & \frac{\partial w}{\partial x} \\
\frac{\partial u}{\partial r} & \frac{\partial v}{\partial r} & \frac{\partial w}{\partial r} \\
\frac{1}{r} \frac{\partial u}{\partial \theta} & \frac{1}{r} \frac{\partial v}{\partial \theta}-\frac{w}{r} & \frac{1}{r} \frac{\partial w}{\partial \theta}+\frac{v}{r}
\end{array}\right\}
\end{aligned}
$$

Transpose of (29b) can be written as (30).

$$
\{\nabla \mathbf{v}\}^{\mathrm{T}}=\left\{\begin{array}{lcc}
\frac{\partial u}{\partial x} & \frac{\partial u}{\partial r} & \frac{1}{r} \frac{\partial u}{\partial \theta} \\
\frac{\partial v}{\partial x} & \frac{\partial v}{\partial r} & \frac{1}{r} \frac{\partial v}{\partial \theta}-\frac{w}{r} \\
\frac{\partial w}{\partial x} & \frac{\partial w}{\partial r} & \frac{1}{r} \frac{\partial w}{\partial \theta}+\frac{v}{r}
\end{array}\right\}
$$

Therefore, stress tensor can be written as in (31).

$$
\tau=\mu\left\{\begin{array}{ccc}
\frac{\partial u}{\partial x}+\frac{\partial u}{\partial x} & \frac{\partial v}{\partial x}+\frac{\partial u}{\partial r} & \frac{\partial w}{\partial x}+\frac{1}{r} \frac{\partial u}{\partial \theta} \\
\frac{\partial u}{\partial r}+\frac{\partial v}{\partial x} & \frac{\partial v}{\partial r}+\frac{\partial v}{\partial r} & \frac{\partial w}{\partial r} \frac{1}{r} \frac{\partial v}{\partial \theta}-\frac{w}{r} \\
\frac{1}{r} \frac{\partial u}{\partial \theta}+\frac{\partial w}{\partial x} & \frac{1}{r} \frac{\partial v}{\partial \theta}-\frac{w}{r}+\frac{\partial w}{\partial r} & \frac{1}{r} \frac{\partial w}{\partial \theta}+\frac{v}{r}+\frac{1}{r} \frac{\partial w}{\partial \theta}+\frac{v}{r}
\end{array}\right\}
$$

$(\tau: \nabla \mathrm{v})$ vector operation is simple but time and space consuming operation. Therefore, it is skipped here. However, some key steps can be summarized as; double dot product by doing dot product two times while paying attention to the order of the terms. This order causes unit vectors interact with partial derivatives and partial derivatives containing azimuthal direction changes unit vectors and creates new terms. The final form of $(\tau: \nabla v)$ is given in (32).

$(\tau: \nabla v)$

$$
=\mu\left(\begin{array}{l}
2\left(\begin{array}{l}
\left(\frac{\partial u}{\partial x}\right)^{2}+\left(\frac{\partial v}{\partial r}\right)^{2}+\left(\frac{1}{r} \frac{\partial w}{\partial \theta}\right)^{2}+\frac{\partial v}{\partial x} \frac{\partial u}{\partial r}+\frac{1}{r} \frac{\partial w}{\partial x} \frac{\partial u}{\partial \theta} \\
+\frac{1}{r} \frac{\partial v}{\partial \theta} \frac{\partial w}{\partial r}+2 \frac{v}{r} \frac{1}{r} \frac{\partial w}{\partial \theta}+\left(\frac{v}{r}\right)^{2}-\frac{w}{r} \frac{\partial w}{\partial r}-\frac{w}{r} \frac{1}{r} \frac{\partial v}{\partial \theta}
\end{array}\right) \\
+\left(\frac{\partial u}{\partial r}\right)^{2}+\left(\frac{1}{r} \frac{\partial u}{\partial \theta}\right)^{2}+\left(\frac{\partial w}{\partial x}\right)^{2}+\left(\frac{\partial w}{\partial r}\right)^{2}+\left(\frac{\partial v}{\partial x}\right)^{2}+\left(\frac{1}{r} \frac{\partial v}{\partial \theta}\right)^{2}+\left(\frac{w}{r}\right)^{2}
\end{array}\right)
$$

Energy equation in terms of temperature in cylindrical coordinates is given explicitly in (33).

$$
\begin{aligned}
& \frac{\partial T}{\partial t}+u \frac{\partial T}{\partial x}+v \frac{\partial T}{\partial r}+\frac{w}{r} \frac{\partial T}{\partial \theta}=\alpha\left(\frac{\partial^{2} T}{\partial x^{2}}+\frac{\partial^{2} T}{\partial r^{2}}+\frac{1}{r^{2}} \frac{\partial^{2} T}{\partial \theta^{2}}+\frac{1}{r} \frac{\partial T}{\partial r}\right)
\end{aligned}
$$

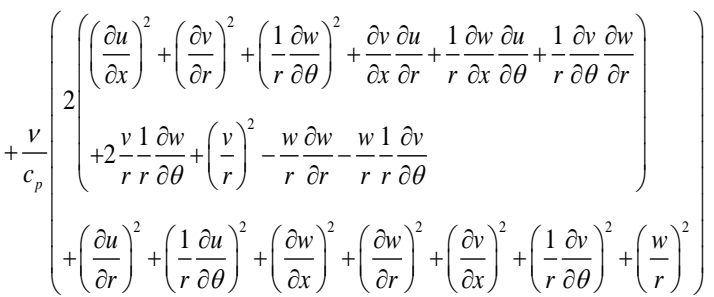

For 2D steady axisymmetric constant viscosity case, governing equations for flow become as following, in respect to their presented order at the beginning of this section.

Continuity equation;

$\frac{\partial u}{\partial x}+\frac{v}{r}+\frac{\partial v}{\partial r}=0$

$x$-momentum;

$u \frac{\partial u}{\partial x}+v \frac{\partial u}{\partial r}=-\frac{1}{\rho} \frac{\partial p}{\partial x}+v\left(\frac{\partial^{2} u}{\partial x^{2}}+\frac{\partial^{2} u}{\partial r^{2}}+\frac{1}{r} \frac{\partial u}{\partial r}\right)$ 
r-momentum;

$u \frac{\partial v}{\partial x}+v \frac{\partial v}{\partial r}=-\frac{1}{\rho} \frac{\partial p}{\partial r}+v\left(\frac{\partial^{2} v}{\partial x^{2}}+\frac{\partial^{2} v}{\partial r^{2}}+\frac{1}{r} \frac{\partial v}{\partial r}-\frac{v}{r^{2}}\right)$

Energy equation for 2D steady axisymmetric constant thermo-physical properties flow can be written with transient term since heat transfer can be transient while flow is steady due to the constant properties assumption.

$\frac{\partial T}{\partial t}+u \frac{\partial T}{\partial x}+v \frac{\partial T}{\partial r}=\alpha\left(\frac{\partial^{2} T}{\partial x^{2}}+\frac{\partial^{2} T}{\partial r^{2}}+\frac{1}{r} \frac{\partial T}{\partial r}\right)$

$+\frac{v}{c_{p}}\left(2\left(\left(\frac{\partial u}{\partial x}\right)^{2}+\left(\frac{\partial v}{\partial r}\right)^{2}+\frac{\partial v}{\partial x} \frac{\partial u}{\partial r}+\left(\frac{v}{r}\right)^{2}\right)+\left(\frac{\partial u}{\partial r}\right)^{2}+\left(\frac{\partial v}{\partial x}\right)^{2}\right)$

$\frac{\partial T}{\partial t}+u \frac{\partial T}{\partial x}+v \frac{\partial T}{\partial r}=\alpha\left(\frac{\partial^{2} T}{\partial x^{2}}+\frac{\partial^{2} T}{\partial r^{2}}+\frac{1}{r} \frac{\partial T}{\partial r}\right)+\frac{\Phi}{\rho c_{p}}$

Final equations are checked by using GOCC.

\subsection{Reynolds decomposition}

The next step is to decompose governing equations into their time average and fluctuating parts and then again taking time average of the whole. The decomposition procedure is known as Reynolds Decomposition (RD). A schematic drawing is given in Figure 2 in order to explain decomposition. Since this work only considers incompressible flows, turbulent velocity component has only two parts; i.e. steady and fluctuating.

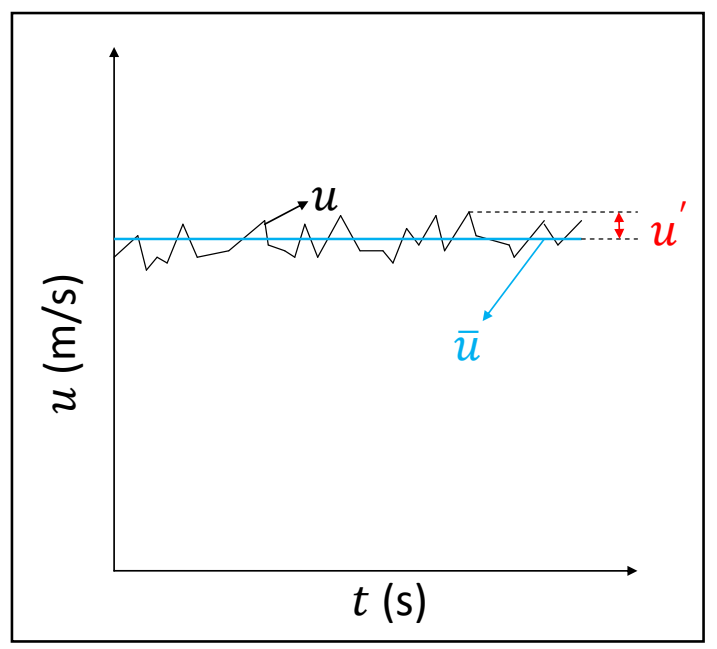

Figure 2. Components of $u$ velocity for decomposition.

$\mathrm{RD}$ rules are given as following:

$$
\bar{u}=\frac{\sum_{t=0}^{t=n} u}{n}
$$

$u^{\prime}=u-\bar{u}$

$\sum_{t=0}^{t=n} u^{\prime}=0$

$\sum_{t=0}^{t=n} \bar{u} u^{\prime}=0$

$\sum_{t=0}^{t=n} u^{\prime} u^{\prime} \neq 0$

$\frac{\sum_{t=0}^{t=n} u^{\prime} u^{\prime}}{n}=\overline{u^{\prime} u^{\prime}} \neq 0$

When governing equations between (34)-(37) are decomposed with RD rules, fluctuating components of the diffusive parts vanish. Convective terms yield new double correlation fluctuating terms and source term in energy equation also yields a fluctuating clone of itself.

Decomposition and time averaging steps of governing equations are given in (44)-(47).

Continuity equation;

$\frac{\partial\left(\bar{u}+u^{\prime}\right)}{\partial x}+\frac{\left(\bar{v}+v^{\prime}\right)}{r}+\frac{\partial\left(\bar{v}+v^{\prime}\right)}{\partial r}=0$

$\overline{\frac{\partial \bar{u}}{\partial x}+\frac{\bar{v}}{r}+\frac{\partial \bar{v}}{\partial r}+\frac{\partial u^{\prime}}{\partial x}+\frac{v^{\prime}}{r}+\frac{\partial v^{\prime}}{\partial r}}=0$

$\frac{\partial \bar{u}}{\partial x}+\frac{\bar{v}}{r}+\frac{\partial \bar{v}}{\partial r}=0$

$x$-momentum;

$$
\begin{aligned}
& \left(\bar{v}+v^{\prime}\right) \frac{\partial\left(\bar{u}+u^{\prime}\right)}{\partial r}+\left(\bar{u}+u^{\prime}\right) \frac{\partial\left(\bar{u}+u^{\prime}\right)}{\partial x} \\
& =-\frac{1}{\rho_{f}} \frac{\partial\left(\bar{p}+p^{\prime}\right)}{\partial x}+v\left[\frac{1}{r} \frac{\partial}{\partial r}\left(r \frac{\partial\left(\bar{u}+u^{\prime}\right)}{\partial r}\right)+\frac{\partial^{2}\left(\bar{u}+u^{\prime}\right)}{\partial x^{2}}\right]
\end{aligned}
$$




$$
\begin{aligned}
& \overline{\bar{v} \frac{\partial \bar{u}}{\partial r}+\bar{u} \frac{\partial \bar{u}}{\partial x}+v^{\prime} \frac{\partial \bar{u}}{\partial r}+u^{\prime} \frac{\partial \bar{u}}{\partial x}} \\
& \overline{+\bar{v} \frac{\partial u^{\prime}}{\partial r}+\bar{u} \frac{\partial u^{\prime}}{\partial x}+v^{\prime} \frac{\partial u^{\prime}}{\partial r}+u^{\prime} \frac{\partial u^{\prime}}{\partial x}}
\end{aligned}
$$

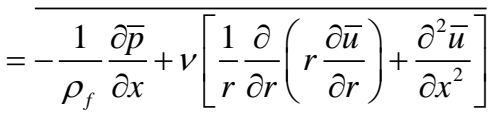

$$
\begin{aligned}
& -\frac{1}{\rho_{f}} \frac{\partial p^{\prime}}{\partial x}+v\left[\frac{1}{r} \frac{\partial}{\partial r}\left(r \frac{\partial u^{\prime}}{\partial r}\right)+\frac{\partial^{2} u^{\prime}}{\partial x^{2}}\right] \\
& \bar{v} \frac{\partial \bar{u}}{\partial r}+\bar{u} \frac{\partial \bar{u}}{\partial x}=-\frac{1}{\rho_{f}} \frac{\partial \bar{p}}{\partial x} \\
& +v\left[\frac{1}{r} \frac{\partial}{\partial r}\left(r \frac{\partial \bar{u}}{\partial r}\right)+\frac{\partial^{2} \bar{u}}{\partial x^{2}}\right] \overline{-v^{\prime} \frac{\partial u^{\prime}}{\partial r}-u^{\prime} \frac{\partial u^{\prime}}{\partial x}} \\
& \bar{v} \frac{\partial \bar{u}}{\partial r}+\bar{u} \frac{\partial \bar{u}}{\partial x}=-\frac{1}{\rho_{f}} \frac{\partial \bar{p}}{\partial x}+v\left[\frac{1}{r} \frac{\partial}{\partial r}\left(r \frac{\partial \bar{u}}{\partial r}\right)+\frac{\partial^{2} \bar{u}}{\partial x^{2}}\right] \\
& -\overline{\left[\left(v^{\prime} \frac{\partial u^{\prime}}{\partial r}=\frac{\partial u^{\prime} v^{\prime}}{\partial r}-u^{\prime} \frac{\partial v^{\prime}}{\partial r}\right)+\left(u^{\prime} \frac{\partial u^{\prime}}{\partial x}=\frac{\partial u^{\prime} u^{\prime}}{\partial x}-u^{\prime} \frac{\partial u^{\prime}}{\partial x}\right)\right]} \\
& \bar{v} \frac{\partial \bar{u}}{\partial r}+\bar{u} \frac{\partial \bar{u}}{\partial x}=-\frac{1}{\rho_{f}} \frac{\partial \bar{p}}{\partial x}+v\left[\frac{1}{r} \frac{\partial}{\partial r}\left(r \frac{\partial \bar{u}}{\partial r}\right)+\frac{\partial^{2} \bar{u}}{\partial x^{2}}\right] \\
& -\overline{\left[\frac{\partial u^{\prime} v^{\prime}}{\partial r}+\frac{\partial u^{\prime} u^{\prime}}{\partial x}-\left(u^{\prime} \frac{\partial v^{\prime}}{\partial r}+u^{\prime} \frac{\partial u^{\prime}}{\partial x}=-\frac{u^{\prime} v^{\prime}}{r}\right)\right]} \\
& \bar{v} \frac{\partial \bar{u}}{\partial r}+\bar{u} \frac{\partial \bar{u}}{\partial x}=-\frac{1}{\rho_{f}} \frac{\partial \bar{p}}{\partial x}+v\left[\frac{1}{r} \frac{\partial}{\partial r}\left(r \frac{\partial \bar{u}}{\partial r}\right)+\frac{\partial^{2} \bar{u}}{\partial x^{2}}\right] \\
& -\left(\frac{\partial \overline{u^{\prime} u^{\prime}}}{\partial x}+\frac{\partial \overline{u^{\prime} v^{\prime}}}{\partial r}+\frac{\overline{u^{\prime} v^{\prime}}}{r}\right)
\end{aligned}
$$

$r$-momentum;

$$
\begin{aligned}
& \left(\bar{v}+v^{\prime}\right) \frac{\partial\left(\bar{v}+v^{\prime}\right)}{\partial r}+\left(\bar{u}+u^{\prime}\right) \frac{\partial\left(\bar{v}+v^{\prime}\right)}{\partial x} \\
& =-\frac{1}{\rho_{f}} \frac{\partial\left(\bar{p}+p^{\prime}\right)}{\partial r}+v\left[\frac{\partial}{\partial r}\left(\frac{1}{r} \frac{\partial\left(r\left(\bar{v}+v^{\prime}\right)\right)}{\partial r}\right)+\frac{\partial^{2}\left(\bar{v}+v^{\prime}\right)}{\partial x^{2}}\right]
\end{aligned}
$$

$$
\begin{aligned}
& \overline{\bar{v} \frac{\partial \bar{v}}{\partial r}+\bar{u} \frac{\partial \bar{v}}{\partial x}+v^{\prime} \frac{\partial \bar{v}}{\partial r}+u^{\prime} \frac{\partial \bar{v}}{\partial x}} \\
& +\bar{v} \frac{\partial v^{\prime}}{\partial r}+\bar{u} \frac{\partial v^{\prime}}{\partial x}+v^{\prime} \frac{\partial v^{\prime}}{\partial r}+u^{\prime} \frac{\partial v^{\prime}}{\partial x} \\
& =-\frac{1}{\rho_{f}} \frac{\partial \bar{p}}{\partial r}+v\left[\frac{\partial}{\partial r}\left(\frac{1}{r} \frac{\partial(r \bar{v})}{\partial r}\right)+\frac{\partial^{2} \bar{v}}{\partial x^{2}}\right] \\
& -\frac{1}{\rho_{f}} \frac{\partial p^{\prime}}{\partial r}+v\left[\frac{\partial}{\partial r}\left(\frac{1}{r} \frac{\partial\left(r v^{\prime}\right)}{\partial r}\right)+\frac{\partial^{2} v^{\prime}}{\partial x^{2}}\right]
\end{aligned}
$$

$$
\begin{aligned}
& \bar{v} \frac{\partial \bar{v}}{\partial r}+\bar{u} \frac{\partial \bar{v}}{\partial x}=-\frac{1}{\rho_{f}} \frac{\partial \bar{p}}{\partial r} \\
& +v\left[\frac{\partial}{\partial r}\left(\frac{1}{r} \frac{\partial(r \bar{v})}{\partial r}\right)+\frac{\partial^{2} \bar{v}}{\partial x^{2}}\right] \overline{-v^{\prime} \frac{\partial v^{\prime}}{\partial r}-u^{\prime} \frac{\partial v^{\prime}}{\partial x}}
\end{aligned}
$$$$
\bar{v} \frac{\partial \bar{v}}{\partial r}+\bar{u} \frac{\partial \bar{v}}{\partial x}=-\frac{1}{\rho_{f}} \frac{\partial \bar{p}}{\partial r}+v\left[\frac{\partial}{\partial r}\left(\frac{1}{r} \frac{\partial(r \bar{v})}{\partial r}\right)+\frac{\partial^{2} \bar{v}}{\partial x^{2}}\right]
$$$$
-\left[\overline{\left(v^{\prime} \frac{\partial v^{\prime}}{\partial r}=\frac{\partial v^{\prime} v^{\prime}}{\partial r}-v^{\prime} \frac{\partial v^{\prime}}{\partial r}\right)+\left(u^{\prime} \frac{\partial v^{\prime}}{\partial x}=\frac{\partial u^{\prime} v^{\prime}}{\partial x}-v^{\prime} \frac{\partial u^{\prime}}{\partial x}\right)}\right]
$$

$$
-\left[\overline{\frac{\partial v^{\prime} v^{\prime}}{\partial r}+\frac{\partial u^{\prime} v^{\prime}}{\partial x}-\left(v^{\prime} \frac{\partial u^{\prime}}{\partial x}-v^{\prime} \frac{\partial v^{\prime}}{\partial r}=-\frac{v^{\prime} v^{\prime}}{r}\right)}\right]
$$$$
\bar{v} \frac{\partial \bar{v}}{\partial r}+\bar{u} \frac{\partial \bar{v}}{\partial x}=-\frac{1}{\rho_{f}} \frac{\partial \bar{p}}{\partial r}+v\left[\frac{\partial}{\partial r}\left(\frac{1}{r} \frac{\partial(r \bar{v})}{\partial r}\right)+\frac{\partial^{2} \bar{v}}{\partial x^{2}}\right]
$$$$
-\left(\frac{\partial \overline{v^{\prime} v^{\prime}}}{\partial r}+\frac{\partial \overline{u^{\prime} v^{\prime}}}{\partial x}+\frac{\overline{v^{\prime} v^{\prime}}}{r}\right)
$$

Only final form of the energy equation is given below since above steps are repeated.

$$
\begin{aligned}
& \frac{\partial \bar{T}}{\partial t}+\bar{v} \frac{\partial \bar{T}}{\partial r}+\bar{u} \frac{\partial \bar{T}}{\partial x}=\alpha\left[\frac{1}{r} \frac{\partial}{\partial r}\left(r \frac{\partial \bar{T}}{\partial r}\right)+\frac{\partial^{2} \bar{T}}{\partial x^{2}}\right] \\
& -\left[\frac{\partial \overline{u^{\prime} T^{\prime}}}{\partial x}+\frac{\partial \overline{v^{\prime} T^{\prime}}}{\partial r}+\frac{\overline{T^{\prime} v^{\prime}}}{r}\right]+\frac{\Phi}{\rho c_{p}}+\frac{\varepsilon}{\rho c_{p}}
\end{aligned}
$$

\subsection{Turbulence modeling}

In order to reduce number of unknowns in RD equations between (44)-(47), turbulence viscosity modeling can be used. For the present case, following modeling statements are written.

$$
\begin{aligned}
& -\overline{u^{\prime} u^{\prime}}=v_{T}\left(\frac{\partial \bar{u}}{\partial x}+\frac{\partial \bar{u}}{\partial x}\right)-\frac{2}{3} k \\
& -\overline{v^{\prime} v^{\prime}}=v_{T}\left(\frac{\partial \bar{v}}{\partial r}+\frac{\partial \bar{v}}{\partial r}\right)-\frac{2}{3} k
\end{aligned}
$$

$-\overline{u^{\prime} v^{\prime}}=-\overline{v^{\prime} u^{\prime}}=v_{T}\left(\frac{\partial \bar{u}}{\partial r}+\frac{\partial \bar{v}}{\partial x}\right)$ 
with $\alpha_{T}=\frac{v_{T}}{\sigma_{T}}$ and $\sigma_{T}=0.9$

$-\overline{u^{\prime} T^{\prime}}=\alpha_{T} \frac{\partial \bar{T}}{\partial x}$

$-\overline{v^{\prime} T^{\prime}}=\alpha_{T} \frac{\partial \bar{T}}{\partial r}$

(48)-(52) is written in (44)-(47) to give modeled equations for turbulent flow. Continuity equation is not written again because it does not contain fluctuating variables. Also, turbulent kinetic energy terms are absorbed in pressure terms. Residual turbulent kinetic energy term in radial momentum equation vanishes by reorganizing the equation. Reorganization also changes some terms due to continuity.

$x$-momentum;

$$
\begin{aligned}
& \bar{v} \frac{\partial \bar{u}}{\partial r}+\bar{u} \frac{\partial \bar{u}}{\partial x}=-\frac{1}{\rho_{f}} \frac{\partial \bar{p}}{\partial x}+v\left[\frac{1}{r} \frac{\partial}{\partial r}\left(r \frac{\partial \bar{u}}{\partial r}\right)+\frac{\partial^{2} \bar{u}}{\partial x^{2}}\right] \\
& +\left\{\begin{array}{l}
\frac{\partial}{\partial x}\left[v_{T}\left(2 \frac{\partial \bar{u}}{\partial x}\right)-\frac{2}{3} k\right]+\frac{\partial}{\partial r}\left[v_{T}\left(\frac{\partial \bar{u}}{\partial r}+\frac{\partial \bar{v}}{\partial x}\right)\right] \\
+\frac{1}{r}\left[v_{T}\left(\frac{\partial \bar{u}}{\partial r}+\frac{\partial \bar{v}}{\partial x}\right)\right]
\end{array}\right\} \\
& \bar{v} \frac{\partial \bar{u}}{\partial r}+\bar{u} \frac{\partial \bar{u}}{\partial x}=-\frac{1}{\rho_{f}} \frac{\partial}{\partial x}\left(\bar{p}+\frac{2}{3} \rho_{f} k \approx \bar{p}\right) \\
& +v\left[\frac{1}{r} \frac{\partial}{\partial r}\left(r \frac{\partial \bar{u}}{\partial r}\right)+\frac{\partial^{2} \bar{u}}{\partial x^{2}}\right]+v_{T}\left[\frac{1}{r} \frac{\partial}{\partial r}\left(r \frac{\partial \bar{u}}{\partial r}\right)+\frac{\partial^{2} \bar{u}}{\partial x^{2}}\right] \\
& +v_{T} \frac{\partial}{\partial x}\left(\frac{\partial \bar{u}}{\partial x}+\frac{\partial \bar{v}}{\partial x}+\frac{\bar{v}}{r}=0\right) \\
& \bar{v} \frac{\partial \bar{u}}{\partial r}+\bar{u} \frac{\partial \bar{u}}{\partial x}=-\frac{1}{\rho_{f}} \frac{\partial \bar{p}}{\partial x}+\left(v+v_{T}\right)\left[\frac{1}{r} \frac{\partial}{\partial r}\left(r \frac{\partial \bar{u}}{\partial r}\right)+\frac{\partial^{2} \bar{u}}{\partial x^{2}}\right]
\end{aligned}
$$

\section{$r$-momentum;}

$$
\begin{gathered}
\bar{v} \frac{\partial \bar{v}}{\partial r}+\bar{u} \frac{\partial \bar{v}}{\partial x}=-\frac{1}{\rho_{f}} \frac{\partial \bar{p}}{\partial r}+v\left[\frac{\partial}{\partial r}\left(\frac{1}{r} \frac{\partial(r \bar{v})}{\partial r}\right)+\frac{\partial^{2} \bar{v}}{\partial x^{2}}\right] \\
+\left\{\begin{array}{l}
\frac{\partial}{\partial r}\left[v_{T}\left(2 \frac{\partial \bar{v}}{\partial r}\right)-\frac{2}{3} k\right]+\frac{\partial}{\partial x}\left[v_{T}\left(\frac{\partial \bar{v}}{\partial x}+\frac{\partial \bar{u}}{\partial r}\right)\right] \\
+\frac{1}{r}\left[v_{T}\left(2 \frac{\partial \bar{v}}{\partial r}\right)-\frac{2}{3} k\right]
\end{array}\right\}
\end{gathered}
$$

$$
\begin{aligned}
& \bar{v} \frac{\partial \bar{v}}{\partial r}+\bar{u} \frac{\partial \bar{v}}{\partial x}=-\frac{1}{\rho_{f}} \frac{\partial}{\partial r}\left(\bar{p}+\frac{2}{3} \rho_{f} k \approx \bar{p}\right) \\
& +v\left[\frac{\partial}{\partial r}\left(\frac{1}{r} \frac{\partial(r \bar{v})}{\partial r}\right)+\frac{\partial^{2} \bar{v}}{\partial x^{2}}\right] \\
& +\left[\begin{array}{l}
2 v_{T} \frac{\partial}{\partial r}\left(\frac{\partial \bar{v}}{\partial r}\right)+v_{T} \frac{\partial}{\partial x} \frac{\partial \bar{v}}{\partial x}+v_{T} \frac{\partial}{\partial x} \frac{\partial \bar{u}}{\partial r} \\
+2 v_{T} \frac{1}{r}\left(\frac{\partial \bar{v}}{\partial r}\right)-\frac{1}{r} \frac{2}{3} k
\end{array}\right]
\end{aligned}
$$

$\bar{v} \frac{\partial \bar{v}}{\partial r}+\bar{u} \frac{\partial \bar{v}}{\partial x}=-\frac{1}{\rho_{f}} \frac{\partial \bar{p}}{\partial r}+v\left[\frac{\partial}{\partial r}\left(\frac{1}{r} \frac{\partial(r \bar{v})}{\partial r}\right)+\frac{\partial^{2} \bar{v}}{\partial x^{2}}\right]$

$+\left[\begin{array}{l}v_{T} \frac{\partial^{2} \bar{v}}{\partial r^{2}}+v_{T} \frac{1}{r}\left(\frac{\partial \bar{v}}{\partial r}\right)+v_{T} \frac{\partial^{2} \bar{v}}{\partial x^{2}} \\ +v_{T} \frac{\partial^{2} \bar{v}}{\partial r^{2}}+v_{T} \frac{1}{r}\left(\frac{\partial \bar{v}}{\partial r}\right)+v_{T} \frac{\partial}{\partial r} \frac{\partial \bar{u}}{\partial x}-\frac{1}{r} \frac{2}{3} k\end{array}\right]$

$\bar{v} \frac{\partial \bar{v}}{\partial r}+\bar{u} \frac{\partial \bar{v}}{\partial x}=-\frac{1}{\rho_{f}} \frac{\partial \bar{p}}{\partial r}+v\left[\frac{\partial}{\partial r}\left(\frac{1}{r} \frac{\partial(r \bar{v})}{\partial r}\right)+\frac{\partial^{2} \bar{v}}{\partial x^{2}}\right]$

$+\left[\begin{array}{l}v_{T} \frac{\partial^{2} \bar{v}}{\partial r^{2}}+v_{T} \frac{1}{r}\left(\frac{\partial \bar{v}}{\partial r}\right)+v_{T} \frac{\partial^{2} \bar{v}}{\partial x^{2}} \\ +v_{T}\left(\frac{\partial}{\partial r}\left(\frac{\bar{v}}{r}+\frac{\partial \bar{v}}{\partial r}\right)+\frac{\bar{v}}{r^{2}}\right)+v_{T} \frac{\partial}{\partial r} \frac{\partial \bar{u}}{\partial x}-\frac{1}{r} \frac{2}{3} k\end{array}\right]$

$\bar{v} \frac{\partial \bar{v}}{\partial r}+\bar{u} \frac{\partial \bar{v}}{\partial x}=-\frac{1}{\rho_{f}} \frac{\partial \bar{p}}{\partial r}+v\left[\frac{\partial}{\partial r}\left(\frac{1}{r} \frac{\partial(r \bar{v})}{\partial r}\right)+\frac{\partial^{2} \bar{v}}{\partial x^{2}}\right]$

$$
+\left[\begin{array}{l}
v_{T} \frac{\partial^{2} \bar{v}}{\partial r^{2}}+v_{T} \frac{1}{r}\left(\frac{\partial \bar{v}}{\partial r}\right)+v_{T} \frac{\partial^{2} \bar{v}}{\partial x^{2}} \\
+v_{T} \frac{\partial}{\partial r}\left(\frac{\bar{v}}{r}+\frac{\partial \bar{v}}{\partial r}+\frac{\partial \bar{u}}{\partial x}=0\right)+v_{T} \frac{\bar{v}}{r^{2}}-\frac{1}{r} \frac{2}{3} k
\end{array}\right]
$$

$\bar{v} \frac{\partial \bar{v}}{\partial r}+\bar{u} \frac{\partial \bar{v}}{\partial x}=-\frac{1}{\rho_{f}} \frac{\partial \bar{p}}{\partial r}+v\left[\frac{\partial}{\partial r}\left(\frac{1}{r} \frac{\partial(r \bar{v})}{\partial r}\right)+\frac{\partial^{2} \bar{v}}{\partial x^{2}}\right]$

$+\left[v_{T}\left(\frac{\partial^{2} \bar{v}}{\partial r^{2}}+\frac{1}{r}\left(\frac{\partial \bar{v}}{\partial r}\right)+\frac{\partial^{2} \bar{v}}{\partial x^{2}}-\frac{\bar{v}}{r^{2}}+\frac{\bar{v}}{r^{2}}+\frac{\bar{v}}{r^{2}}\right)-\frac{1}{r} \frac{2}{3} k\right]$

$\bar{v} \frac{\partial \bar{v}}{\partial r}+\bar{u} \frac{\partial \bar{v}}{\partial x}=-\frac{1}{\rho_{f}} \frac{\partial \bar{p}}{\partial r}+v\left[\frac{\partial}{\partial r}\left(\frac{1}{r} \frac{\partial(r \bar{v})}{\partial r}\right)+\frac{\partial^{2} \bar{v}}{\partial x^{2}}\right]$ 


$$
\begin{aligned}
& \bar{v} \frac{\partial \bar{v}}{\partial r}+\bar{u} \frac{\partial \bar{v}}{\partial x}=-\frac{1}{\rho_{f}} \frac{\partial \bar{p}}{\partial r} \\
& +\left(v+v_{T}\right)\left[\frac{\partial}{\partial r}\left(\frac{1}{r} \frac{\partial(r \bar{v})}{\partial r}\right)+\frac{\partial^{2} \bar{v}}{\partial x^{2}}\right] \\
& +\left[2 v_{T} \frac{1}{r}\left(\frac{\bar{v}}{r}\right)-\frac{1}{r} \frac{2}{3} k\right] \\
& \bar{v} \frac{\partial \bar{v}}{\partial r}+\bar{u} \frac{\partial \bar{v}}{\partial x}=-\frac{1}{\rho_{f}} \frac{\partial \bar{p}}{\partial r} \\
& +\left(v+v_{T}\right)\left[\frac{\partial}{\partial r}\left(\frac{1}{r} \frac{\partial(r \bar{v})}{\partial r}\right)+\frac{\partial^{2} \bar{v}}{\partial x^{2}}\right] \\
& +\left[2 v_{T} \frac{1}{r}\left(-\frac{\partial \bar{u}}{\partial x}-\frac{\partial \bar{v}}{\partial r}\right)-\frac{1}{r} \frac{2}{3} k\right] \\
& \bar{v} \frac{\partial \bar{v}}{\partial r}+\bar{u} \frac{\partial \bar{v}}{\partial x}=-\frac{1}{\rho_{f}} \frac{\partial \bar{p}}{\partial r} \\
& +\left(v+v_{T}\right)\left[\frac{\partial}{\partial r}\left(\frac{1}{r} \frac{\partial(r \bar{v})}{\partial r}\right)+\frac{\partial^{2} \bar{v}}{\partial x^{2}}\right] \\
& +\left[-\frac{1}{r}\left(2 v_{T} \frac{\partial \bar{u}}{\partial x}+2 v_{T} \frac{\partial \bar{v}}{\partial r}\right)-\frac{1}{r} \frac{2}{3} k\right] \\
& \bar{v} \frac{\partial \bar{v}}{\partial r}+\bar{u} \frac{\partial \bar{v}}{\partial x}=-\frac{1}{\rho_{f}} \frac{\partial \bar{p}}{\partial r} \\
& +\left(v+v_{T}\right)\left[\frac{\partial}{\partial r}\left(\frac{1}{r} \frac{\partial(r \bar{v})}{\partial r}\right)+\frac{\partial^{2} \bar{v}}{\partial x^{2}}\right] \\
& +\left[-\frac{1}{r}\left(-u^{\prime} u^{\prime}+\frac{2}{3} k-v^{\prime} v^{\prime}+\frac{2}{3} k\right)-\frac{1}{r} \frac{2}{3} k\right] \\
& \bar{v} \frac{\partial \bar{v}}{\partial r}+\bar{u} \frac{\partial \bar{v}}{\partial x}=-\frac{1}{\rho_{f}} \frac{\partial \bar{p}}{\partial r} \\
& +\left(v+v_{T}\right)\left[\frac{\partial}{\partial r}\left(\frac{1}{r} \frac{\partial(r \bar{v})}{\partial r}\right)+\frac{\partial^{2} \bar{v}}{\partial x^{2}}\right] \\
& +\left[\frac{1}{r}\left(u^{\prime} u^{\prime}+v^{\prime} v^{\prime}=2 k\right)-\frac{1}{r} 2 k\right] \\
& \bar{v} \frac{\partial \bar{v}}{\partial r}+\bar{u} \frac{\partial \bar{v}}{\partial x}=-\frac{1}{\rho_{f}} \frac{\partial \bar{p}}{\partial r} \\
& +\left(v+v_{T}\right)\left[\frac{\partial}{\partial r}\left(\frac{1}{r} \frac{\partial(r \bar{v})}{\partial r}\right)+\frac{\partial^{2} \bar{v}}{\partial x^{2}}\right]
\end{aligned}
$$

Above procedure is valid for the energy equation. Therefore, only final form is given below.

$$
\begin{aligned}
& {\left[\frac{\partial \bar{T}}{\partial t}+\bar{v} \frac{\partial \bar{T}}{\partial r}+\bar{u} \frac{\partial \bar{T}}{\partial x}\right]} \\
& =\left(\frac{k}{\rho c_{p}}+\alpha_{T}\right)\left[\frac{1}{r} \frac{\partial}{\partial r}\left(r \frac{\partial \bar{T}}{\partial r}\right)+\frac{\partial^{2} \bar{T}}{\partial x^{2}}\right]+\frac{\Phi}{\rho c_{p}}+\frac{\varepsilon}{\rho c_{p}}
\end{aligned}
$$

A way of calculating turbulent viscosity is using $k-\epsilon$ turbulence modeling approach. The relation between turbulent kinetic energy, dissipation and turbulent viscosity is given in (56).

$$
\begin{aligned}
& v_{T}=C_{\mu} \frac{k^{2}}{\varepsilon} \\
& C_{\mu}=0.09
\end{aligned}
$$

Derivation of equations for turbulent kinetic energy and its dissipation is explained in $[4,6]$. In order to obtain turbulent kinetic energy equation, momentum equations before decomposition are multiplied by velocity components in their respected direction. Obtained equations are decomposed and time averaged. Then those equations are put together to form a single equation. Similarly, decomposed and time averaged momentum equations are multiplied by time averaged velocity components in their direction. Those equations are also added together. This second summation forms kinetic energy equation of the mean flow. When second summation is subtracted from first summation, turbulent kinetic energy is obtained. Dissipation equation requires additional and different approaches. Obtained turbulent kinetic energy and dissipation equations are modeled by using turbulent viscosity approach. This is required because those equations have triple correlations of fluctuating terms. This complex process can lead practitioners to mistakes. Instead, vector form from [1] is used again in order to write $\mathrm{k}$ and $\epsilon$ equations in cylindrical coordinates.

$$
\nabla \square(\rho v k)=\nabla\left(\left(\mu+\frac{\mu_{T}}{\sigma_{k}}\right) \nabla k\right)+\mu_{T} \Phi-\rho \varepsilon
$$

$\nabla \square(\rho \mathrm{v} \varepsilon)=\nabla\left\{\left(\left(\mu+\frac{\mu_{T}}{\sigma_{\varepsilon}}\right) \nabla \varepsilon\right)+C_{\varepsilon, 1} \frac{\varepsilon}{k} \mu_{T} \Phi-C_{\varepsilon, 2} \rho \frac{\varepsilon^{2}}{k}\right.$

Model constants are $\sigma_{k}=1, \sigma_{\varepsilon}=1.3, C_{\varepsilon, 1}=1.44$ and $C_{\varepsilon, 2}=1.92$ 
The final forms of turbulent kinetic energy and dissipation equations in cylindrical coordinates are given in (60) and (61).

Turbulent kinetic energy model equation;

$\bar{u} \frac{\partial k}{\partial x}+\bar{v} \frac{\partial k}{\partial r}=\left(\frac{v_{T}}{\sigma_{k}}\right)\left[\frac{\partial^{2} k}{\partial x^{2}}+\frac{1}{r} \frac{\partial}{\partial r}\left(r \frac{\partial k}{\partial r}\right)\right]$

$+v_{T}\left\{\left(\frac{\partial \bar{u}}{\partial r}+\frac{\partial \bar{v}}{\partial x}\right)^{2}+2\left[\left(\frac{\partial \bar{u}}{\partial x}\right)^{2}+\left(\frac{\bar{v}}{r}\right)^{2}+\left(\frac{\partial \bar{v}}{\partial r}\right)^{2}\right]\right\}-\varepsilon$

Turbulent kinetic energy dissipation equation;

$$
\begin{aligned}
& \bar{u} \frac{\partial \varepsilon}{\partial x}+\bar{v} \frac{\partial \varepsilon}{\partial r}=\left(\frac{v_{T}}{\sigma_{\varepsilon}}\right)\left[\frac{\partial^{2} \varepsilon}{\partial x^{2}}+\frac{1}{r} \frac{\partial}{\partial r}\left(r \frac{\partial \varepsilon}{\partial r}\right)\right] \\
& +C_{\varepsilon, 1} \frac{\varepsilon}{k} v_{T}\left\{\left(\frac{\partial \bar{u}}{\partial r}+\frac{\partial \bar{v}}{\partial x}\right)^{2}+2\left[\left(\frac{\partial \bar{u}}{\partial x}\right)^{2}+\left(\frac{\bar{v}}{r}\right)^{2}+\left(\frac{\partial \bar{v}}{\partial r}\right)^{2}\right]\right\} \\
& -C_{\varepsilon, 2} \frac{\varepsilon^{2}}{k}
\end{aligned}
$$

Standard $k-\epsilon$ turbulence model necessitates wall functions since isotropic turbulence assumption is not valid near walls. Viscous sub-layer and logarithmic layers are solved by means of wall functions as source terms. Related standard wall functions are written below after the arrangement for cylindrical coordinates.

$x$-momentum wall function that models wall stress

$$
-\frac{\tau_{w}}{\rho_{f}} A_{w}=-\frac{C_{\mu}^{1 / 4} k^{1 / 2} \bar{u}}{\frac{1}{\kappa} \ln \left(E \frac{C_{\mu}^{1 / 4} k^{1 / 2}\left(r_{w}-r\right)}{v}\right)} r \Delta x
$$

where $\kappa=0.41$ and $E=9.793$.

Wall function for $k$ equation production and dissipation terms;

$$
\begin{aligned}
& \left(P_{k}-\varepsilon\right) \Delta V \\
& =\left[\frac{\tau_{w}}{\rho_{f}\left(r_{w}-r\right)} \bar{u}-\frac{C_{\mu}^{3 / 4} k^{3 / 2} \ln \left(E \frac{C_{\mu}^{1 / 4} k^{1 / 2}\left(r_{w}-r\right)}{v}\right)}{\kappa\left(r_{w}-r\right)}\right] r \Delta r \Delta x
\end{aligned}
$$

Wall function for $\epsilon$ value at the calculation node;

$$
\varepsilon=\frac{C_{\mu}^{3 / 4} k^{3 / 2}}{\kappa\left(r_{w}-r\right)}
$$

Generally numerical grid is arranged for flow solution. Accordingly, the node at which wall function of the energy equation is solved can lie in linear or logarithmic layers. Therefore, two types of wall functions are given below for the energy equation. These wall functions calculate the wall heat flux.

For logarithmic layer;

$$
\frac{q_{w} A_{w}}{\rho c_{p}}=\frac{\left(T_{w}-T\right) C_{\mu}^{1 / 4} k^{1 / 2}}{\sigma_{T}\left[\frac{1}{\kappa} \ln \left(E \frac{C_{\mu}^{1 / 4} k^{1 / 2}\left(r_{w}-r\right)}{v}\right)+P\right]} r \Delta x
$$

$P$ in (65) is a function described in [8],[9] and it is given below.

$$
P=9.24\left[\left(\frac{\mathrm{Pr}}{\sigma_{T}}\right)^{3 / 4}-1\right]\left[1+0.28 e^{-0.007\left(\frac{\mathrm{Pr}}{\sigma_{T}}\right)}\right]
$$

Wall function for the energy equation in linear region is given below.

$\frac{q_{w} A_{w}}{\rho c_{p}}=\frac{\left(T_{w}-T\right) C_{\mu}^{1 / 4} k^{1 / 2}}{\operatorname{Pr} \frac{C_{\mu}^{1 / 4} k^{1 / 2}\left(r_{w}-r\right)}{v}} r \Delta x$

\subsection{Nondimensionalization}

The last step is making equations non-dimensional. Following parameters are proposed for this process.

$$
\begin{aligned}
& u^{*}=\frac{\bar{u}}{u_{0}} \\
& v^{*}=\frac{\bar{v}}{u_{0}}
\end{aligned}
$$

$T^{*}=\frac{\bar{T}-T_{0}}{T_{1}-T_{0}}$

$$
x^{*}=\frac{x}{2 r_{w}}
$$

$$
r^{*}=\frac{r}{2 r_{w}}
$$

$$
d^{*}=d_{w f}=\frac{d}{2 r_{w}}
$$

$$
k_{w f}=\frac{k_{w}}{k_{f}}
$$


$\alpha_{w f}=\frac{\alpha_{w}}{\alpha_{f}}$

$t^{*}=\frac{t \alpha_{f}}{4 r_{w}^{2}}=\mathrm{Fo}$

$P^{*}=\frac{P}{\frac{1}{2} \rho u_{0}^{2}}$

$k^{*}=\frac{k}{\frac{u_{0}^{2}}{2}}$

$\varepsilon^{*}=\frac{\varepsilon}{\frac{u_{0}^{3}}{2 r_{w}}}$

$v_{T}^{*}=\frac{v_{T}}{u_{0} 2 r_{w}}$

$\alpha_{T}^{*}=\frac{\alpha_{T}}{u_{0} 2 r_{w}}$

$q_{w}^{*}=\frac{q_{w} 2 r_{w}}{k\left(T_{1}-T_{0}\right)} \Rightarrow$

$q_{w}^{*}=\frac{\operatorname{Pe}\left(T_{w}^{*}-T_{f_{p}}^{*}\right) C_{\mu}^{1 / 4} k^{* / 2}}{\sqrt{2} \sigma_{T}\left[\frac{1}{\kappa} \ln \left(E \frac{C_{\mu}^{1 / 4} k^{* / 2} \operatorname{Re}\left(0.5-r^{*}\right)}{\sqrt{2}}\right)+P\right]}$

$\operatorname{Re}=\frac{u_{0} 2 r_{w}}{v}$

$\operatorname{Pr}=\frac{\mu c_{p}}{k}=\frac{v \rho c_{p}}{k}$

$\mathrm{Pe}=\frac{2 r_{w} u_{0} \rho c_{p}}{k}=\frac{2 r_{w} u_{0}}{\alpha}$

$\mathrm{Ec}=\frac{u_{0}^{2}}{c_{p}\left(T_{1}-T_{0}\right)}$

Dimensionless governing equations are given in the following by using above dimensionless parameters. Their order is; continuity equation, $x$ momentum and $r$ momentum equations, turbulent kinetic energy and turbulent kinetic energy dissipation equations, energy equation and wall functions.

$\frac{\partial v^{*}}{\partial r^{*}}+\frac{v^{*}}{r^{*}}+\frac{\partial u^{*}}{\partial x^{*}}=0$

$$
\begin{aligned}
& v^{*} \frac{\partial u^{*}}{\partial r^{*}}+u^{*} \frac{\partial u^{*}}{\partial x^{*}}=-\frac{1}{2} \frac{\partial p^{*}}{\partial x^{*}} \\
& +\left(\frac{1}{\operatorname{Re}}+v_{T}^{*}\right)\left[\frac{1}{r^{*}} \frac{\partial}{\partial r^{*}}\left(r^{*} \frac{\partial u^{*}}{\partial r^{*}}\right)+\frac{\partial^{2} u^{*}}{\partial x^{* 2}}\right] \\
& v^{*} \frac{\partial v^{*}}{\partial r^{*}}+u^{*} \frac{\partial v^{*}}{\partial x^{*}}=-\frac{1}{2} \frac{\partial p^{*}}{\partial r^{*}} \\
& +\left(\frac{1}{\operatorname{Re}}+v_{T}^{*}\right)\left[\frac{\partial}{\partial r^{*}}\left(\frac{1}{r^{*}} \frac{\partial r^{*} v^{*}}{\partial r^{*}}\right)+\frac{\partial^{2} v^{*}}{\partial x^{* 2}}\right] \\
& u^{*} \frac{k^{*}}{\partial x^{*}}+v^{*} \frac{\partial k^{*}}{\partial r^{*}}=\left(\frac{v_{T}^{*}}{\sigma_{k}}\right)\left[\frac{\partial^{2} k^{*}}{\partial x^{* 2}}+\frac{1}{r^{*}} \frac{\partial}{\partial r^{*}}\left(r^{*} \frac{\partial k^{*}}{\partial r^{*}}\right)\right] \\
& +2 v_{T}^{*}\left\{\left(\frac{\partial u^{*}}{\partial r^{*}}+\frac{\partial v^{*}}{\partial x^{*}}\right)^{2}+2\left[\left(\frac{\partial u^{*}}{\partial x^{*}}\right)^{2}+\left(\frac{v^{*}}{r^{*}}\right)^{2}+\left(\frac{\partial v^{*}}{\partial r^{*}}\right)^{2}\right]\right\}-2 \varepsilon^{*} \\
& u^{*} \frac{\partial \varepsilon^{*}}{\partial x^{*}}+v^{*} \frac{\partial \varepsilon^{*}}{\partial r^{*}}\left(\frac{v_{T}^{*}}{\sigma_{\varepsilon}}\right)\left[\frac{\partial^{2} \varepsilon^{*}}{\partial x^{* 2}}+\frac{1}{r^{*}} \frac{\partial}{\partial r^{*}}\left(r^{*} \frac{\partial \varepsilon^{*}}{\partial r^{*}}\right)\right] \\
& +2 C_{\varepsilon, 1} \frac{\varepsilon^{*}}{k^{*}} v_{T}^{*}\left\{\left(\frac{\partial u^{*}}{\partial r^{*}}+\frac{\partial v^{*}}{\partial x^{*}}\right)^{2}+2\left[\left(\frac{\partial u^{*}}{\partial x^{*}}\right)^{2}+\left(\frac{v^{*}}{r^{*}}\right)^{2}+\left(\frac{\partial v^{*}}{\partial r^{*}}\right)^{2}\right]\right\} \\
& -2 C_{\varepsilon, 2} \frac{\varepsilon^{*}}{k^{*}} \\
& v_{T}^{*}=\frac{C_{\mu}}{4} \frac{k^{* 2}}{\varepsilon^{*}}
\end{aligned}
$$

$\frac{1}{\operatorname{Pe}} \frac{\partial T^{*}}{\partial t^{*}}+v^{*} \frac{\partial T^{*}}{\partial r^{*}}+u^{*} \frac{\partial T^{*}}{\partial x^{*}}$

$=\left(\frac{1}{\mathrm{Pe}}+\alpha_{T}^{*}\right)\left[\frac{1}{r^{*}} \frac{\partial}{\partial r^{*}}\left(r^{*} \frac{\partial T^{*}}{\partial r^{*}}\right)+\frac{\partial^{2} T^{*}}{\partial x^{* 2}}\right]$

$+\frac{\mathrm{Ec}}{\mathrm{Re}} \Phi^{*}+\mathrm{Ec} \varepsilon^{*}$

$-\tau_{w}^{*} A_{w}^{*}=-\frac{C_{\mu}^{1 / 4} k^{* / 2} u^{*}}{\frac{\sqrt{2}}{\kappa} \ln \left(E C_{\mu}^{1 / 4} k^{* / 2} \frac{\operatorname{Re}}{\sqrt{2}}\left(0.5-r^{*}\right)\right)} r^{*}(\Delta x)^{*}$

$2\left(P_{k}^{*}-\varepsilon^{*}\right) \Delta V^{*}=2\left[\begin{array}{c}\frac{C_{\mu}^{1 / 4} k^{\mu / 2} u^{22}}{\frac{\sqrt{2}}{\kappa} \ln \left(E C_{\mu}^{1 / 4} k^{2 / 2} \frac{\mathrm{Re}}{\sqrt{2}}\left(0.5-r^{*}\right)\right)\left(0.5-r^{*}\right)} \\ -\frac{C_{\mu}^{3 / 4} k^{3 / 2} \ln \left(E C_{\mu}^{1 / 4} k^{4 / 2} \frac{\mathrm{Re}}{\sqrt{2}}\left(0.5-r^{*}\right)\right)}{2^{3 / 2} \kappa\left(0.5-r^{*}\right)}\end{array}\right] r^{*}(\Delta r)^{*}(\Delta x)^{*}$

$\varepsilon^{*}=\frac{C_{\mu}^{3 / 4} k^{* 3 / 2}}{2^{3 / 2} \kappa\left(0.5-r^{*}\right)}$ 


$$
\begin{aligned}
& \frac{q_{w}^{*} A_{w}^{*}}{\operatorname{Pe}}=\frac{\left(T_{w}^{*}-T^{*}\right) C_{\mu}^{1 / 4} k^{* / / 2}}{\sqrt{2} \sigma_{T}\left[\frac{1}{\kappa} \ln \left(E \frac{C_{\mu}^{1 / 4} k^{* / 2} \operatorname{Re}\left(0.5-r^{*}\right)}{\sqrt{2}}\right)+P\right]^{*}(\Delta x)^{*}} \\
& \frac{q_{w}^{*} A_{w}^{*}}{\operatorname{Pe}}=\frac{\left(T_{w}^{*}-T^{*}\right)}{\operatorname{Pr} \operatorname{Re}\left(0.5-r^{*}\right)} r^{*}(\Delta x)^{*}
\end{aligned}
$$

Finally, dimensionless wall coordinate is derived from $y^{+}$by using $k-\epsilon$ turbulence model.

$$
y^{*}=\frac{C_{\mu}^{1 / 4} k^{1 / 2}\left(r_{w}-r\right)}{v}=\frac{C_{\mu}^{1 / 4} k^{* / 2} \operatorname{Re}\left(0.5-r^{*}\right)}{\sqrt{2}}
$$

In the next section, this formulation flow and its implications are evaluated.

\section{Implications}

The first point to be addressed in the governing equations written in cylindrical coordinates is additional terms containing $1 / r$. From geometrical and physics point of view, these terms emerge due to increasing surface area of a differential volume as radius increases. In order to conserve mass, for instance, velocity in radial direction should decrease; otherwise mass would be generated due to increasing arc area. This phenomenon is tried to be illustrated by Figure 3 . Figure 3 is a pseudoschematic arrangement in which flow enters the domain having only axial velocity component and exits with only radial velocity component. This fictional domain has nothing to do with the derivational procedure of the previous section. It is drawn only for physical reasoning.

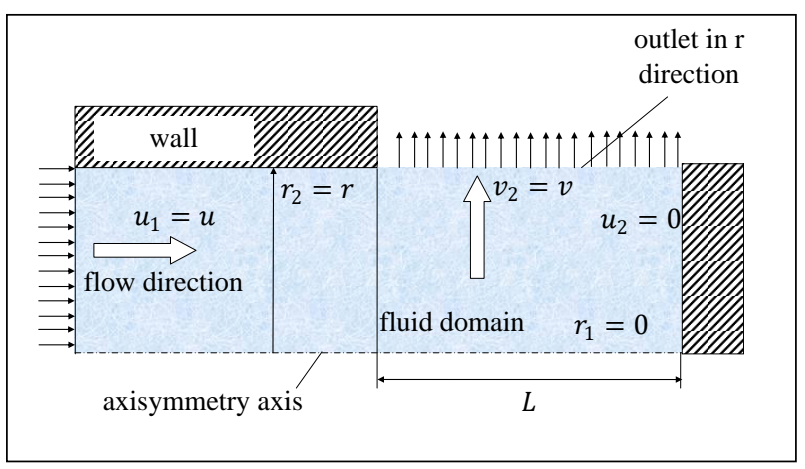

Figure 3. Schematics for illustrating $1 / r$ terms by continuity.
For a 2D axisymmetric control volume as depicted in Figure 3, continuity can be written by a sequence of mathematical operations given in (100)-(107). Since mass is conserved, inlet and outlet mass flow rates are equal. Therefore, the relation between axial and radial velocities can be expressed as (102). Taking partial spatial derivatives of velocity components and summing them for conservation of mass flow rate should necessitate a deficit as $\Delta V$ since there should be additional term for continuity. After reorganization, the additional term is found as in (107).

$$
\begin{aligned}
& \dot{m}=\rho V A \\
& \frac{\rho u \pi r^{2}}{2 \pi}=\rho v\left[\left(\frac{2 \pi}{2 \pi}\right) r L\right]
\end{aligned}
$$

$\frac{u r}{2}=v L$

$\frac{\partial u}{\partial x}=\frac{u_{2}-u_{1}}{L}=\frac{0-u}{L}=-\frac{u}{L}=-\frac{\frac{2 v L}{r}}{L}=-\frac{2 v}{r}$

$\frac{\partial v}{\partial r}=\frac{v_{2}-v_{1}}{r_{2}-r_{1}}=\frac{v-0}{r-0}=\frac{v}{r}$

$\frac{\partial u}{\partial x}+\frac{\partial v}{\partial r}+\Delta V=0$

$-\frac{2 v}{r}+\frac{v}{r}+\Delta V=0$

$\Delta V=\frac{v}{r}$

Next issue is the appearance of Reynolds stresses (turbulent stresses) and turbulence modeling in momentum equations. Reynolds stresses have negative sign comparing with viscous diffusion of momentum while modeled turbulence terms have positive sign. In order to explain this phenomenon an illustrative example is presented. A probe immersed in developed steady turbulent pipe flow at the symmetry axis would measure an axial velocity as in Figure 4. Of course, this schematic illustration is for identifying components of the measured velocity and roles of turbulence and turbulence modeling on them. Axial velocity, fluctuating with time for steady regime has two 
components; time averaged constant component and fluctuating component that has different values by time. Turbulent stresses or Reynolds stresses disturb layers of laminar flow, decreasing effects of molecular viscosity towards symmetry axis. However, these stresses have their energy cost and hence they have contribution to pressure drop of the flow. As local velocity increases, turbulence also increases and acts as a regulator. This is resulted with a relatively lower developed velocity at symmetry axis comparing with the laminar flow case. Modeled terms induce this effect with a different mechanism. They reduce flow energy with a pseudo viscosity (turbulence viscosity) that changes its value locally. In other words, Reynolds stresses decrease the effects of diffusion of laminar stresses while modeled turbulence decreases momentum flux and hence flow energy.

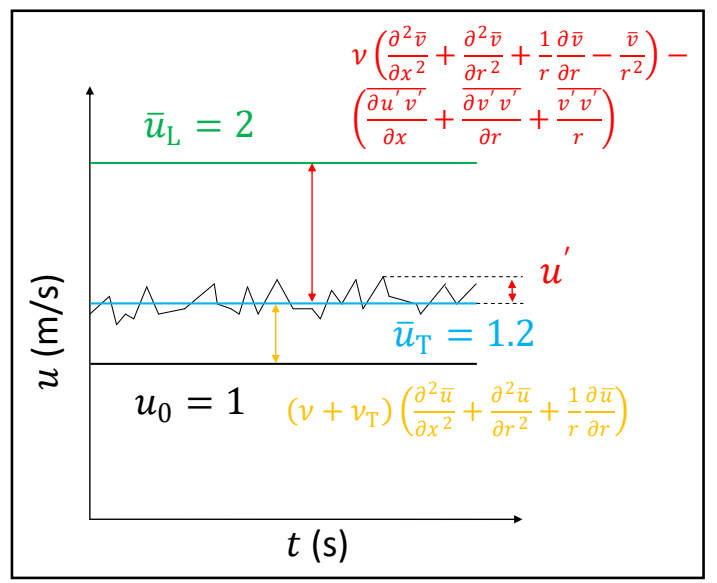

Figure 4. Schematics for dimensionless axial velocity component at symmetry axis for a pipe flow.

A similar projection can be made for pipe flow axial velocity profile in radial direction (Figure 5 ). While turbulent stresses make velocity profile less parabolic, turbulence modeling extract energy from uniform velocity profile to reach the same powerlaw profile.

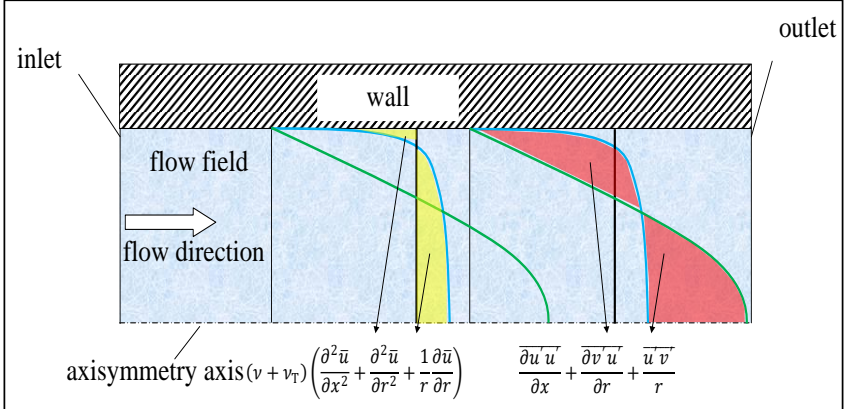

Figure 5. Schematics for velocity profiles, Reynolds stresses and modelled turbulence terms.

Next focus is on non-dimensionalization. For making governing equations non-dimensional, simple scales are preferred, aiming Reynolds number the only parameter for momentum equations. For hydrodynamic investigation, only length and velocity scales are used as pipe radius and inlet velocity respectively. This leads to Reynolds number and dimensionless turbulent viscosity in the momentum equations. Since turbulent viscosity is calculated, only independent parameter to be investigated is Reynolds number.

Temperature in energy equation is not just dimensionless but also normalized. This is desired because it limits the investigation to an interval where all assumptions such as constant viscosity are valid, and no additional measures are taken.

The scales for nondimensionalization lead to $P e$ and $E c / R e$ in energy equation. $E c / R e$ appears as a parameter for viscous dissipation and $E c$ is for turbulent dissipation. Normally, Ec number is used as a parameter for viscous heating and Mach number $(M a)$ is also stated as a limit to evaluation of viscous heating versus $E c$ number. The nondimensionalization process intrinsically yields $E c / R e$ but physically this combination induces effects of solid boundaries and viscosity. Therefore, it is planned to conduct a CFD analysis in future to see viscous heating for various values of $E c / R e$.

\section{Conclusion and Remarks}

In this work, governing equations for CFD are written in cylindrical coordinates explicitly by using vector forms as starting point and vector operations 
for extracting them into final forms. Important points in vector operations special to cylindrical coordinates are emphasized. Means of reorganization are explained. Equations are then simplified for 2D axisymmetric incompressible turbulent flow. Some parameters are proposed for making equations non-dimensional. Following remarks can be given:

- Writing governing equations explicitly for a specific coordinate system seems to be applied best by using vector forms of the equations as a starting point.

- Special care should be given to vector operators and operations since they change according to coordinate system.

- Continuity equation in the chosen coordinate system is devised several times in order to reorganize equations. Some terms in the reorganized equations constitute continuity equation and hence they are equal to " 0 ". The terms that emerge during reorganization of Reynolds Decomposition equations in order to have double correlation of fluctuating velocity components are cancelled by this way. Some of the additional terms that emerge with the application of turbulent viscosity approach in order to replace fluctuating terms are also cancelled by this way.

- Making governing equations non-dimensional needs careful decisions in order to have dimensionless numbers of interest in the governing equations. Also, it is recommended to use primitive constants such as inlet velocity.

- Using normalized temperature instead of nondimensional temperature is necessary to stay in the interval of assumptions such as constant viscosity.

- Although turbulence modeling and Reynolds stresses lead to the same velocity profiles, their mechanism are different, and they effect on different phenomena.

\section{Acknowledgement}

Present content is partly taken and partly derived from Ph.D. thesis of Canli (2020).

\section{References}

Anderson, D.A., Tannehill, J.C., Pletcher, R.H., 1984. Computational Fluid Mechanics and Heat Transfer. USA, Hemisphere publishing corporation, 181-235.

Anonymous, 2009. ANSYS FLUENT 12.0. Theory Guide. ANSYS, Inc., 4:1-4:58.

Canli, E., 2020. Numerical solution of transient conjugated heat transfer in thick walled pipes with turbulent flow. Ph.D. Thesis, Selcuk University Institute of Sciences, Konya, Turkey, 153.

Happel, J., Brenner, H., 2012. Low Reynolds number hydrodynamics: with special applications to particulate media. 1, Springer Science \& Business Media, 474-524.

Jayatilleke, C.L.V., 1966. The influence of Prandtl number and surface roughness on the resistance of the laminar sub-layer to momentum and heat transfer. PhD Thesis, Imperial College of Science and Technology, London, UK, 271.

Launder, B.E., Spalding, D.B, 1974. The numerical computation of turbulent flows. Computer Methods in Applied Mechanics and Engineering, 3(2), 269-289.

Moukalled, F., Mangani, L., Darwish, M., 2016. The finite volume method in computational fluid Dynamics. 113, Berlin, Germany, Springer, 9-82.

Patankar, S., 1980. Numerical heat transfer and fluid flow. London, CRC Press, 25-139.

Tennekes, H., Lumley, J., 1972. A first course in turbulence. The MIT Press, 149-248.

Versteeg, H.K., Malalasekera, W., 2007. An introduction to computational fluid dynamics: the finite volume method. Pearson education, 40-281.

\section{Nomenclature}

\footnotetext{
$A_{w} \quad$ Wall surface area $\left(\mathrm{m}^{2}\right)$

$\alpha \quad$ Thermal diffusivity coefficient $\left(\mathrm{m}^{2} / \mathrm{s}\right)$

$\alpha_{T} \quad$ Turbulent thermal diffusivity coefficient $\left(\mathrm{m}^{2} / \mathrm{s}\right)$
} 


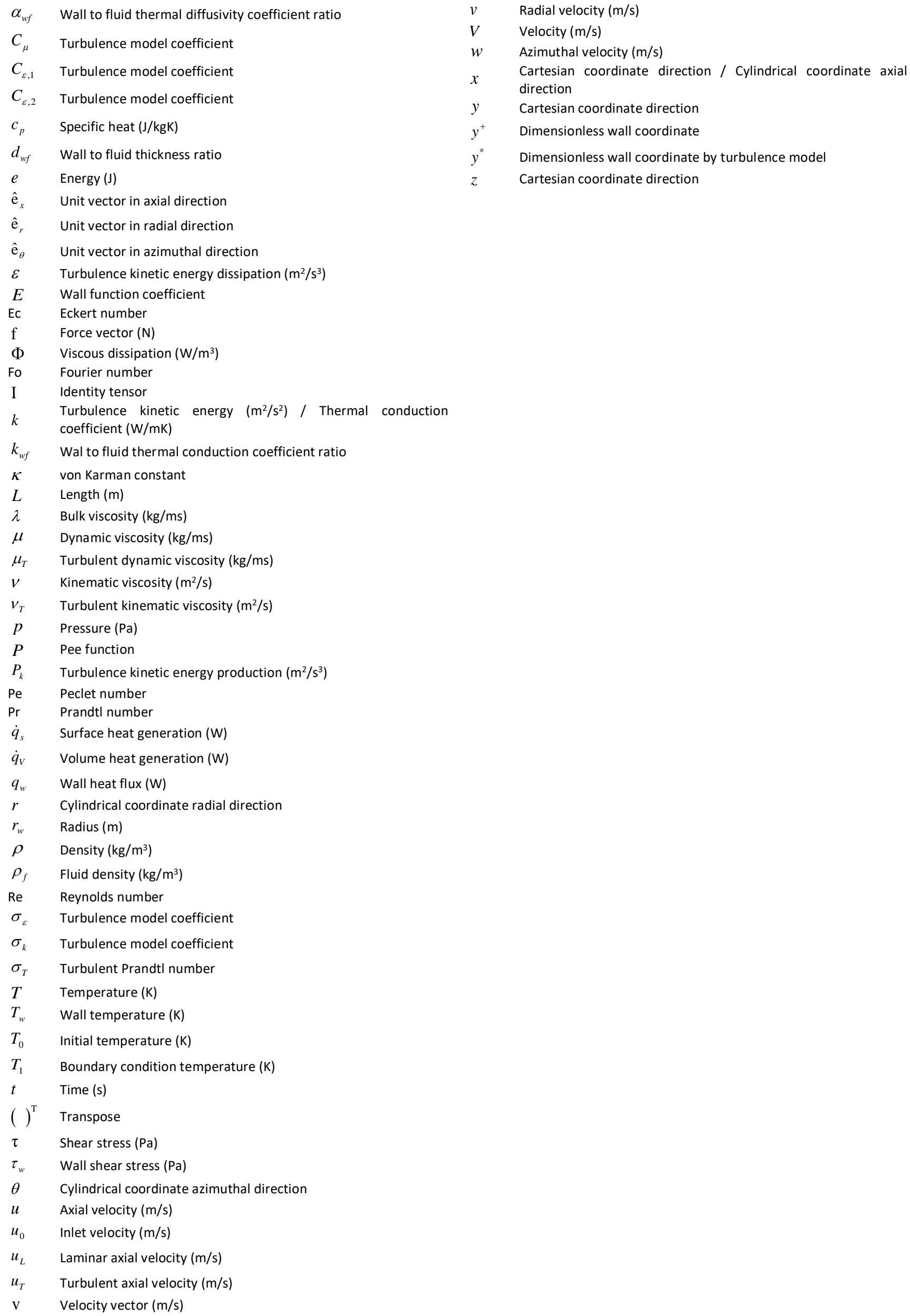

$v \quad$ Radial velocity $(\mathrm{m} / \mathrm{s})$

$V \quad$ Velocity $(\mathrm{m} / \mathrm{s})$

$w \quad$ Azimuthal velocity $(\mathrm{m} / \mathrm{s})$

Cartesian coordinate direction / Cylindrical coordinate axial direction

y Cartesian coordinate direction

$y^{+} \quad$ Dimensionless wall coordinate

$y^{*} \quad$ Dimensionless wall coordinate by turbulence model

$z \quad$ Cartesian coordinate direction 